\title{
Potential Energy Curves \& Material Properties
}

\author{
Devarakonda Annapurna Padmavathi
}

Chemistry Department, Post Graduate College of Science, Saifabad Osmania University, Hyderabad, India.

Email: dapadma@rediffmail.com

Received August $6^{\text {th }}, 2010$; revised December $16^{\text {th }}, 2010$; accepted January $19^{\text {th }}, 2011$.

\begin{abstract}
Potential energy curves govern the properties of materials. A critical analysis of the potential energy curve helps better understand the properties of the material. Potential energy curve and in turn the properties of any material depend on the composition, bonding, crystal structure, their mechanical processing and microstructure. The type, strength, and directionality of atomic bonding controls the structure and material properties viz., melting temperature, thermal expansion, elastic stiffness, electrical properties, ductility and toughness etc. This paper attempts to bring out the correlation between the potential energy curves with the properties of materials.
\end{abstract}

Keywords: Potential Energy Curves, Material Properties

\section{Introduction}

Properties are the way the material responds to the environment and external forces. Mechanical properties respond to mechanical forces, strength, etc. Electrical and magnetic properties deal with their response to electrical and magnetic fields, conductivity etc. Thermal properties are related to transmission of heat and heat capacity.

With the aid of potential energy curve(s), this paper intends to see the response of the material, from the atomic and subatomic particle arrangement, towards external forces. Interatomic forces present in atomic bonding is reflected in the potential energy curves which in turn help predict many physical properties namely melting temperature, elasticity, thermal expansion, and strength of materials. Choice of a material for a specific purpose can be made from the materials performance under different conditions and is reflected in potential energy curves.

Content in the paper is organized with the description of atomic structure and bonding, first, followed by analysis of how it gets reflected in the potential energy curve and subsequently proceeds to bring the relation of material properties with potential energy curves.

\section{Atomic Structure and Bonding}

All the elements that exist are classified according to electronic configuration in the periodic table. Electrons in atoms have discrete energy states and tend to occupy lowest available energy levels. The electronic structure of atoms governs their interaction with other atoms.
Filled outer shells result in a stable configuration as in noble inert gases. Atoms with incomplete outer shells strive to reach this noble gas configuration by sharing or transferring electrons among each other for maximal stability.

There are two main types of bonding: 1) Primary bonding 2) Secondary bonding

1) Primary bonding results from the electron sharing or transfer. There are three types of primary bonding viz., ionic, covalent, and metallic (24-240 kcal/mol) [1].

In ionic bonding, atoms behave like either positive or negative ions, and are bound by Coulomb forces. A large difference in electronegativity is required for an ionic bond to be formed. The ionic bonding is nondirectional, i.e., the magnitude of the bond is equal in all directions. In ceramics, bonding is predominantly ionic. They are usually combinations of metals or semiconductors with oxygen, nitrogen or carbon (oxides, nitrides, and carbides). Ionic materials are hard and brittle due to electrically charged nature of component ions. And, furthermore they are electrical and thermal insulators due to absence of large number of free electrons. (Examples: glass, porcelain, examples of other ceramic materials range from household items to high performance combustion engines which utilize both metals and ceramics.)

Covalent Bonding: In covalent bonding, electrons are shared between the molecules, to saturate the valency. Covalent bonds are highly directional. The simplest example is the $\mathrm{SiO}_{2}$ molecule. Their electrical properties depend strongly on minute proportions of contaminants 
(Examples: $\mathrm{Si}, \mathrm{Ge}, \mathrm{GaAs}$ ).

Metallic bonding: In metals, valence electrons are detached from atoms, and spread in an 'electron sea' that "glues" the ions together. Metals and alloys exhibit four characteristic properties namely good ductility, high thermal conductivity, high electrical conductivity and metallic lustre due to their free electrons. Metallic bonding is nondirectional and is rather insensitive to structure. As a result high ductility is observed in metals where the "bonds" do not "break" when atoms are rearranged examples of metals with typical metallic bonding: $\mathrm{Cu}, \mathrm{Al}$, $\mathrm{Au}, \mathrm{Ag}$, etc. Transition metals (Fe, Ni, etc.) form mixed bonds, comprising of metallic and covalent bonds involving their 3d-electrons. As a result the transition metals are more brittle (less ductile) than $\mathrm{Au}$ or $\mathrm{Cu}$.

2) Secondary bonding: There is no electron transfer or sharing in secondary bonding. Secondary bonding also called as van der Waals bonding, is much weaker as compared to the primary bonding and results from interaction of atomic or molecular dipoles. Range of energy is $<24 \mathrm{kcal} / \mathrm{mol}$.

Large difference in electronegativities between atoms, result in asymmetrical arrangement of positively and negatively charged regions $\left(\mathrm{HCl}, \mathrm{H}_{2} \mathrm{O}\right)$ causing permanent dipole moments. These polar molecules can induce dipoles in adjacent non-polar molecules and bond is formed due to the attraction between the permanent and induced dipoles. Even in electrically symmetric molecules/atoms (like $\mathrm{H}_{2}, \mathrm{Cl}_{2}$ ) an electric dipole can be induced by fluctuations of electron density distribution. Fluctuating electric field in one atom is felt by the electrons of an adjacent atom, and induces a dipole momentum in the other. This bond due to fluctuating induced dipoles is the weakest (inert gases, $\mathrm{H}_{2}, \mathrm{Cl}_{2}$ ).

The strength of the secondary bonding depends on strength of the dipole. Examples include permanent dipoles (polar molecules- $\mathrm{H}_{2} \mathrm{O}, \mathrm{HCl} . .$. ), fluctuating induced dipoles (inert gases, $\mathrm{H}_{2}, \mathrm{Cl}_{2}$ ), dipole-induced dipole bonds and induced dipole-induced dipole interactions.

\subsection{Bonding Forces and Energies}

Physical properties are predicted based on interatomic forces that bind the atoms together. Consider the interaction of two isolated atoms as they are getting closer from an infinite separation. At large distances interactions are negligible but interactions grow up as they approach each other. These forces are of two types attractive force $\left(\mathrm{F}_{\mathrm{A}}\right)$ and repulsive force $\left(\mathrm{F}_{\mathrm{R}}\right)$ and the magnitude of each is a function of interatomic distance. The origin of the attractive part, depends on the particular type of bonding. The repulsion between atoms, when they are brought close to each other, is related to the
Pauli principle: when the electronic clouds surrounding the atoms start to overlap, the energy of the system increases abruptly.

The net force $\mathrm{F}$ is sum of both attractive and repulsive components. When $\mathrm{F}_{\mathrm{A}}$ and $\mathrm{F}_{\mathrm{R}}$ balance, or become equal, there is no net force i.e., $\mathrm{F}_{\mathrm{A}}+\mathrm{F}_{\mathrm{R}}=0$. Then a state of equilibrium exists. This corresponsds to equilibrium spacing as indicated in Figure 1. Sometimes it is more convenient to work with potential energies between two atoms instead of forces.

Mathematically energy $(E)$ and force $(F)$ are related as

$$
E=\int F d r
$$

for atomic systems. At equilibrium spacing $r_{\mathrm{o}}$, net force is zero and net energy corresponds to minimum energy $E_{\mathrm{o}}$ [1]. When there are more than two atoms, force and energy interactions among many atoms have to be considered. The minimum energy $E_{\mathrm{o}}$ is the binding energy required to separate two atoms from their equilibrium spacing to an infinite distance apart.

Figure 2 illustrates simple potential energy curves. The energy $E_{0}$, shape and depth of the curve defines various properties. The curves indicate the strength of the bond based on the depth of the potential well. The more deep the well, the more stable is the molecule, and a shallower potential well indicates the molecule has low

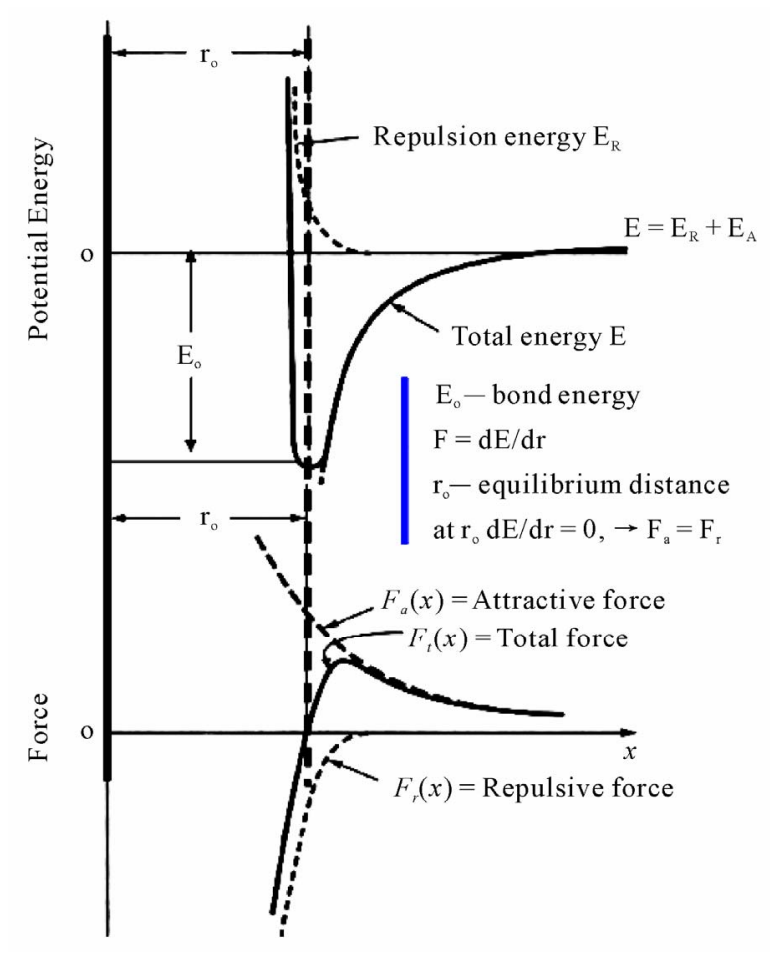

Figure 1. A typical potential well indicating bonding energies $E_{0}$ and forces for two interacting atoms. 

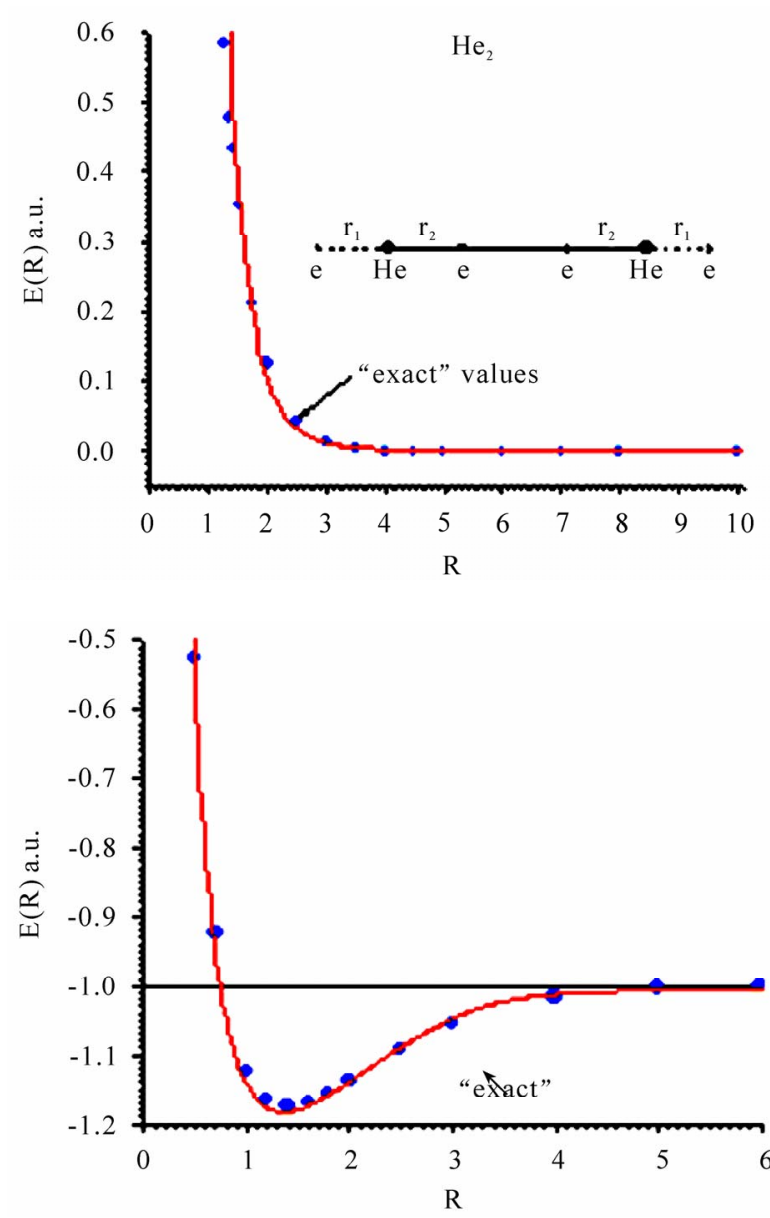

Figure 2. Calculated quantum mechanical potential energy curves of $\mathrm{He}_{2}$ and $\mathrm{H}_{2}$.

dissociation energy. The shallow potential energy curve of helium states that the forces that bind are very weak. Almost a zero $E_{o}$ value indicates the instability of the molecule. Very small bonding energy $E_{o}$ of hydrogen states that gaseous state is favored.

Table 1 gives list of bonding energies and melting tenperatures for various bond types. Increase in depth of the potential well, increases the melting temperature $T_{m}$ (Figure 3(b)). Molecules with large bonding energies have high melting temperatures generally these exist as solids. As the depth of the potential well decreases. the molecules move from solid state to gaseous state [3].

\section{Analysis of Potential Energy Curves}

\subsection{Packing of Crystal Structures and Their Influence on Bonding Energies}

Different atoms based on their nature, arrange themselves in different crystalline forms. The order in which atoms associate with neighbors, determine the bonding energy, the shape and depth of the potential well.
Table 1. Bonding energies and melting temperatures for various substances.

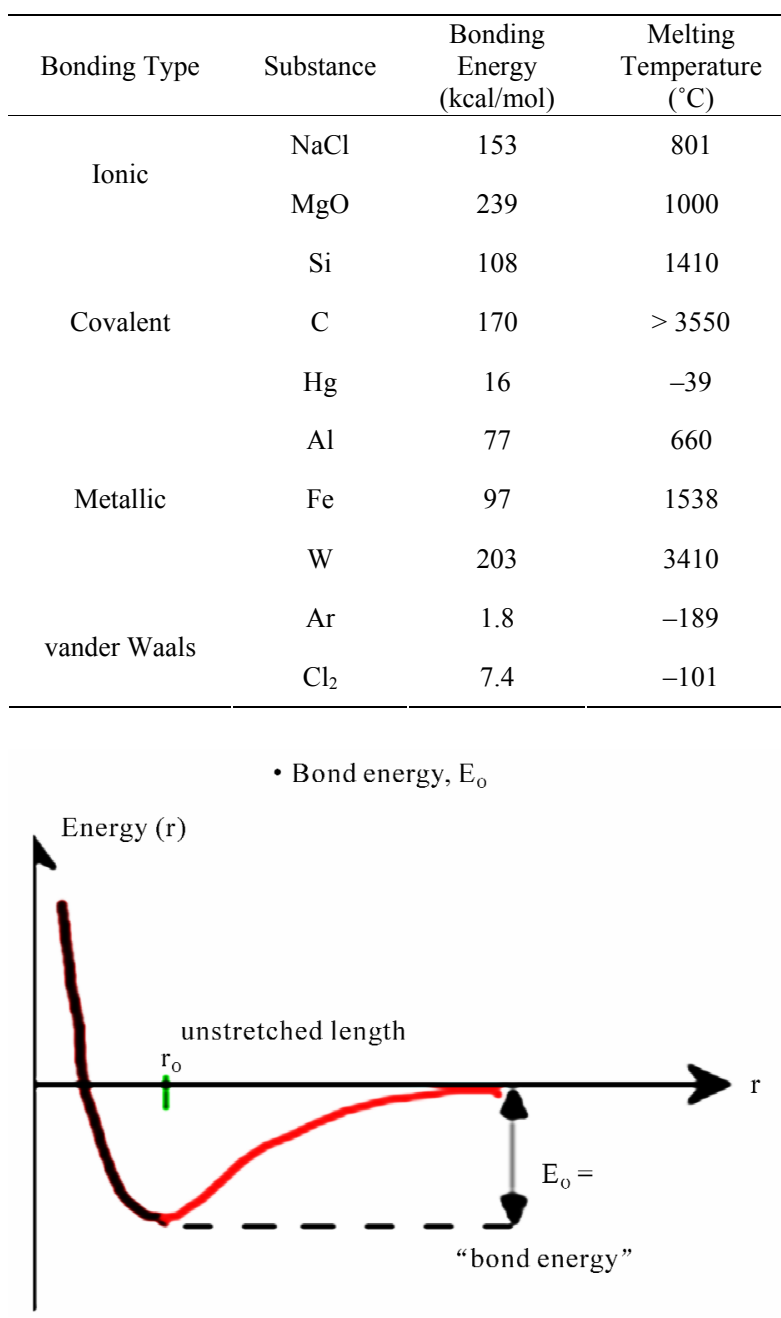

(a)

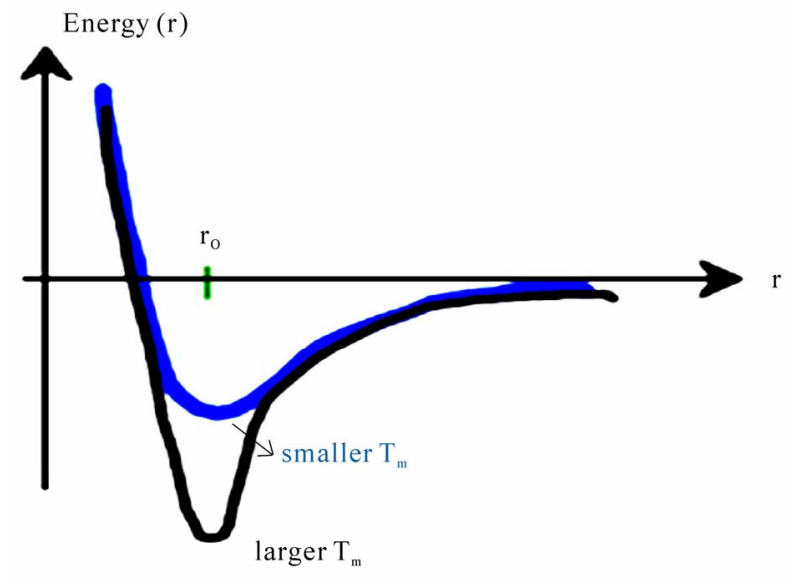

(b)

Figure 3. (a) A typical potential energy curve; (b) Change in shape of the well with temperature. 
Non-crystalline solids lack a systematic and regular arrangement of atoms over relatively large atomic distances. This disordered or random packing of atoms changes the force that binds the neighbouring atoms, and hence the bonding energy $E_{0}$ of neighbouring atoms is different (Figure 4(a)). Due to the variation in neighbouring bond lengths, materials density decreases which has an influence on material properties.

In crystal structures with long range order atoms are positioned in a repititive 3-dimensional pattern in which each atom is bonded to its nearest neighbouring atoms with similar force. As a result, equilibrium bond length $r_{o}$ and bond energy $E_{0}$ remains same between any two neigbouring atoms (Figure 4(b)). And hence materials with high ordered packing have good density and hence good strength [2].

Different ordered packing results in different crystalline patterns. The type of packing, nearest neighbour bonding and crystal structure decide the properties of substances. For ex., pure Mg hexagonal closely packed crystal is more brittle than $\mathrm{Al}$ a face centered cubic crystal due to less number of slip planes and hence undergoes fracture at lower degrees of deformation (Figure 5).

\subsection{Mechanical Properties}

How materials deform (elongate, compress, twist) or break as a function of applied load, time, temperature, and other conditions is described by mechanical properties

The standard language to discuss mechanical properties of materials is in terms of Hooke's law. In this law, stress ' $\sigma$ ' and strain ' $\varepsilon$ ' are related to each other by the equation

$$
\sigma=E \varepsilon
$$

where $\mathrm{E}$ is the modulus of Elasticity or Young's Modulus (Figure 6(a)).

Hooke's law allows one to compare specimens of different cross sectional area $A_{0}$ and different length $L_{0}$. This equation can also be written in terms of force $\mathrm{F}$ as

$$
\frac{F}{A_{o}}=E \frac{\Delta L}{L_{0}}
$$

In the elastic limit Modulus of elasticity $\mathrm{E}$ is the slope of the stress $\left(F / A_{0}\right)$ versus strain $\left(\Delta L / L_{0}\right)$ curve (Figure 6(b)). Higher the modulus of elasticity higher is the stiffness of the bond. After the stress is removed, if the material returns to the dimension it had before the loading, it is elastic deformation. If the material does not return to its previous dimension it is referred as plastic deformation.

On an atomic scale, macroscopic elastic strain is mani-
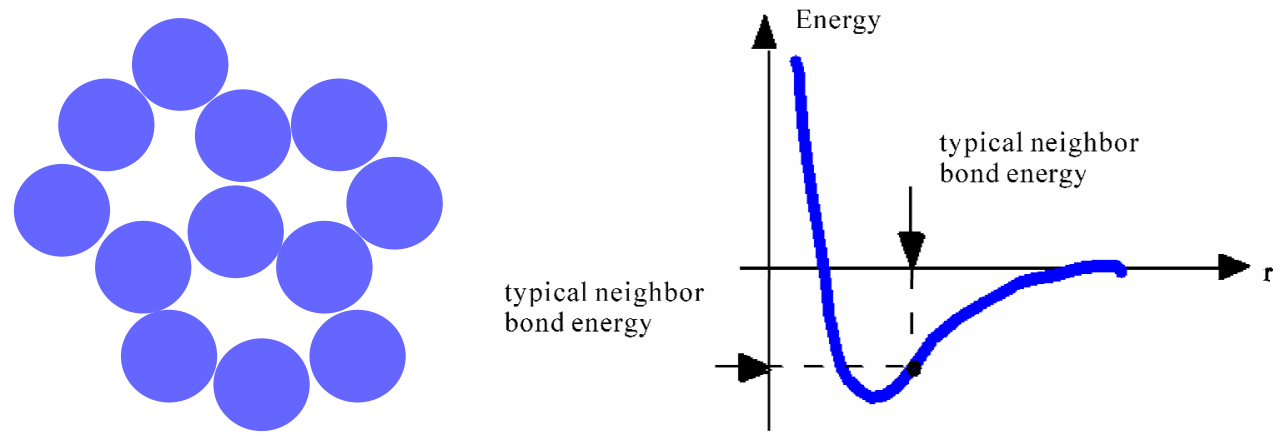

(a)
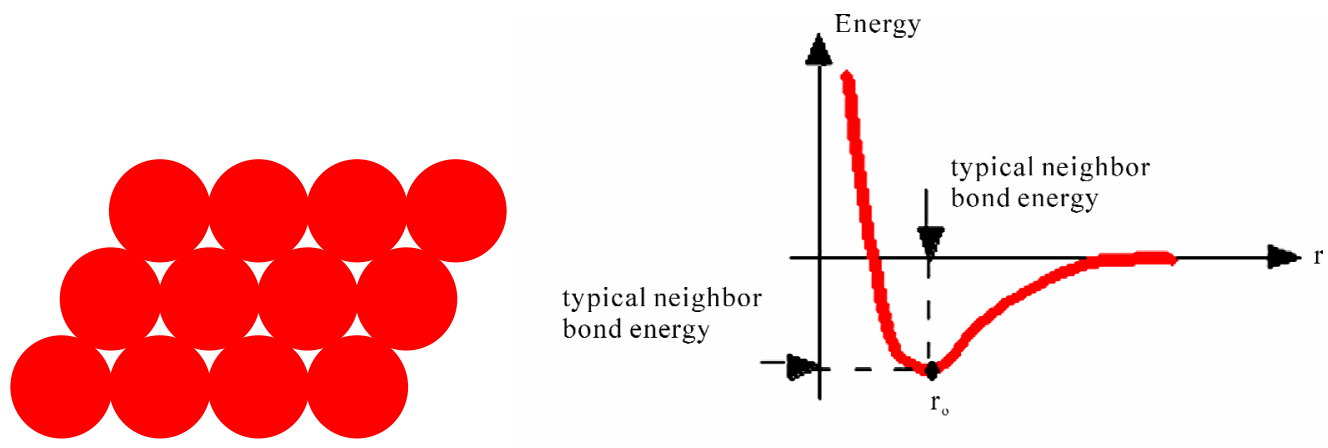

(b)

Figure 4. (a) Random packing of atoms and the corresponding potential energy curve; (b) Dense ordered packing of atoms and the corresponding potential energy curve. 


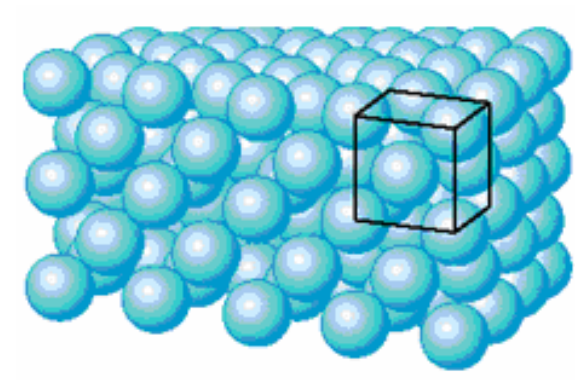

(a)

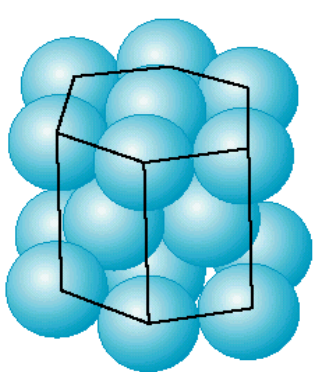

(b)

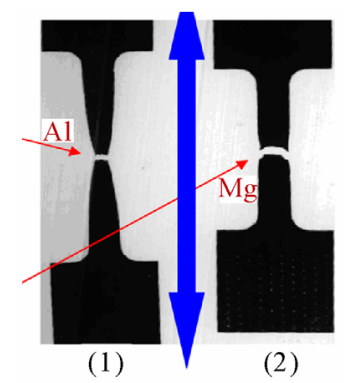

(c)

Figure 5. Packing of atoms in (a) $\mathrm{Al}$, (b) Mg and (c) Relation between packing and fracture.

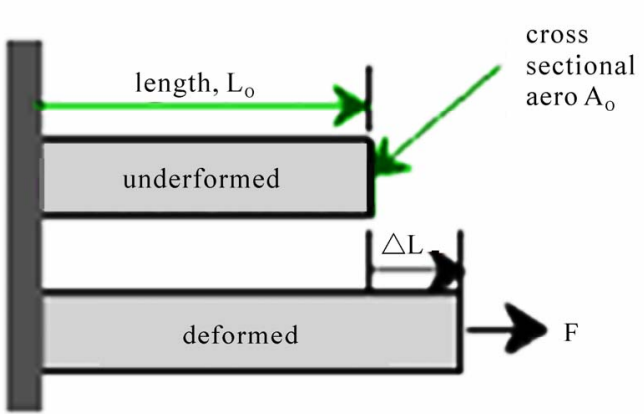

(a)

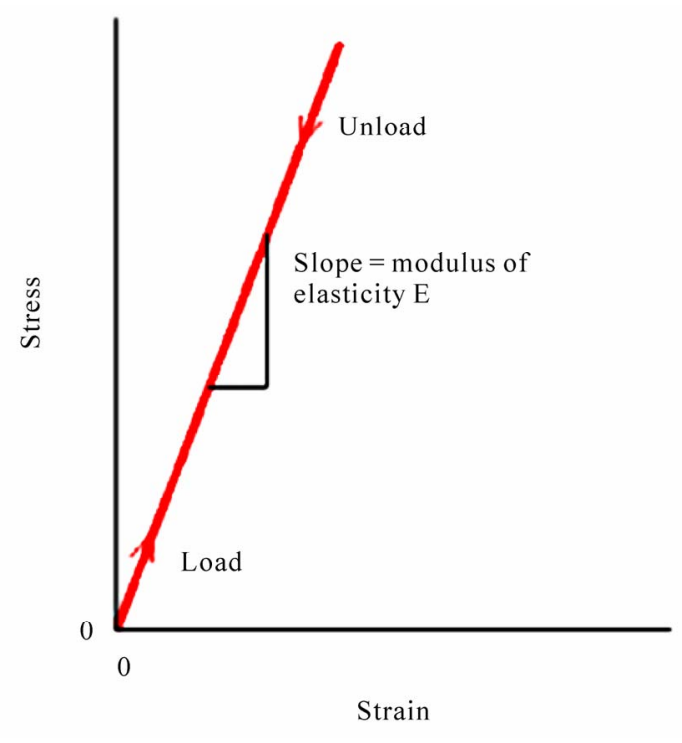

(b)

Figure 6. (a) Variation of stress with strain; (b) Linearity of stress strain relation.

fested as small changes in the interatomic spacing and the stretching of interatomic bonds. As a consequence, the magnitude of modulus of elasticity is a measure of the resistance to separation of adjacent atoms i.e., interatomic bonding forces.

Modulus of eleasticity $\mathrm{E}$ is proportional to slope of the force versus interatomic separation curve (Figure 7(a)) at equilibrium spacing. Slope of the curve at $r=r_{0}$ position is steep for very stiff materials and shallower for flexible materials $[2,3]$.

The energy interatomic distance curve, Figure 7(b) illustrates that as modulus of elasticity E decreases, energy minima decreases and hence the strength of the bond. Values of modulus of eleasticity $\mathrm{E}$ are highest for ceramics, higher for metals and lower for polymers which is a direct consequence of the different types of atomic bonding.

Another measured mechanical property is yield strength. This is the level of stress above which a material begins to show permanent deformation. From an atomic perspective, plastic deformation corresponds to the breaking of bonds with original atom neighbors and then formation of bonds with new ones as large number of molecules move relative to one another. In plastic deformation, upon removal of stress the atoms do not return to their original positions.

Low yield strength corresponds to the inability of the molecule to regain its initial state, which corresponds to low elastic modulus and hence low bonding energy in the potential energy curve.

Metals have high yield strength but for ceramics yield strength is hard to measure, as since in tension fracture occurs before it yields.

\subsection{Thermal Properties}

Response of a material to the application of heat is often studied in terms of heat capacity, thermal expansion and thermal conductivity. In solids the principle mode of thermal energy assimilation is through increase in vibration energy of atoms. The vibrations of adjacent atoms are coupled based on the nature of atomic bonding leading to lattice waves termed phonons which transfer energy through material.

Most solids expand on heating and contract on cooling. Thermal expansion results in an increase in the average distance between atoms. When the temperature changes, 


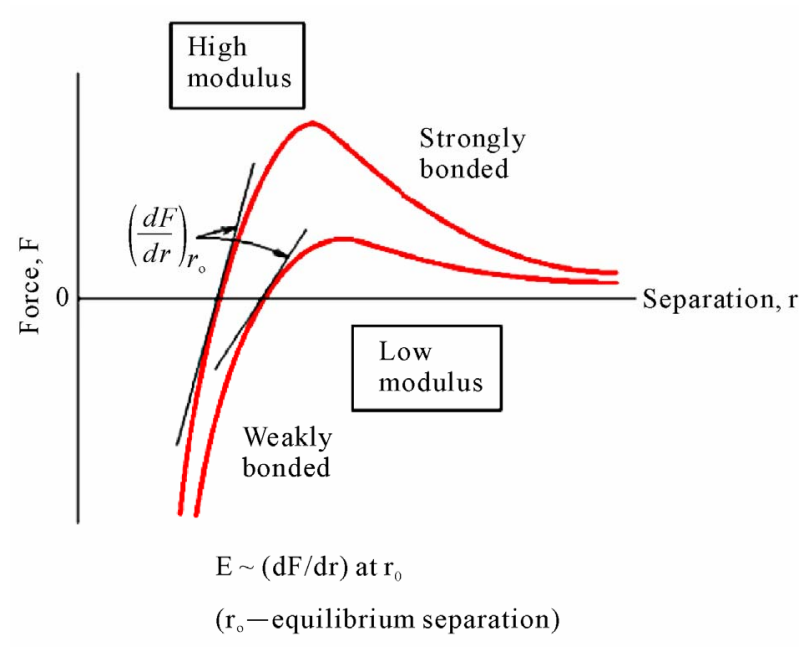

(a)

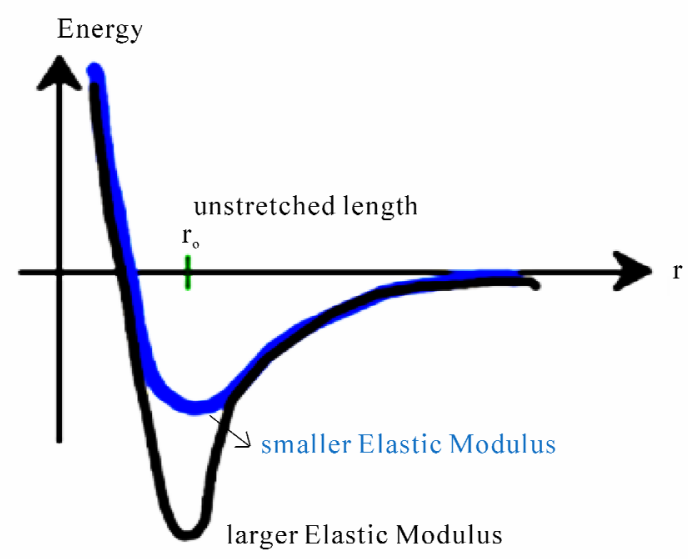

(b)

Figure 7. (a) The force-distance curve for two materials, showing the relationship between atomic bonding and the modulus of elasticity, a steep $\mathrm{dF} / \mathrm{dr}$ slope gives a high modulus; (b) Potential curve with variation of inter atomic distance and energy.

the amount by which a material changes its dimensions in length, is given by linear coefficient of thermal expansion ' $\alpha$ '.

$$
\frac{\Delta L}{L_{0}}=\alpha\left(T_{2}-T_{1}\right)
$$

Linear coefficient of thermal expansion ' $\alpha$ ' of the material can be correlated with the shape of the curve. The trough in the potential energy curve, corresponds to the equilibrium interatomic spacing at $0 \mathrm{~K}$.

Heating to successively higher temperatures (Figure 8(a)) $T_{1}$ to $T_{5}$ raises the vibrational energy. At each temperature, the width of the curve is proportional to the amplitude of thermal vibrations for an atom, and the average interatomic distance is represented by the mean

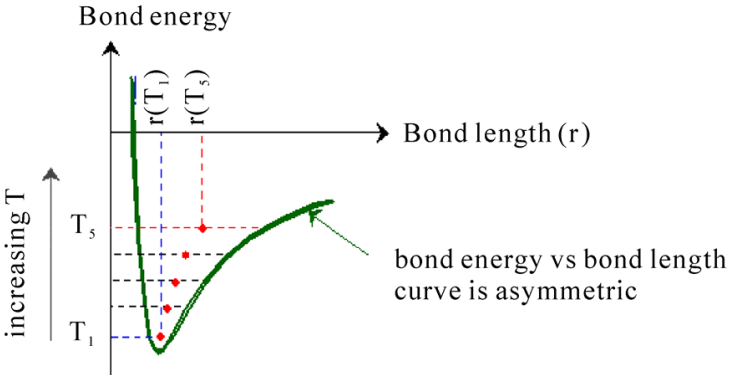

(a)

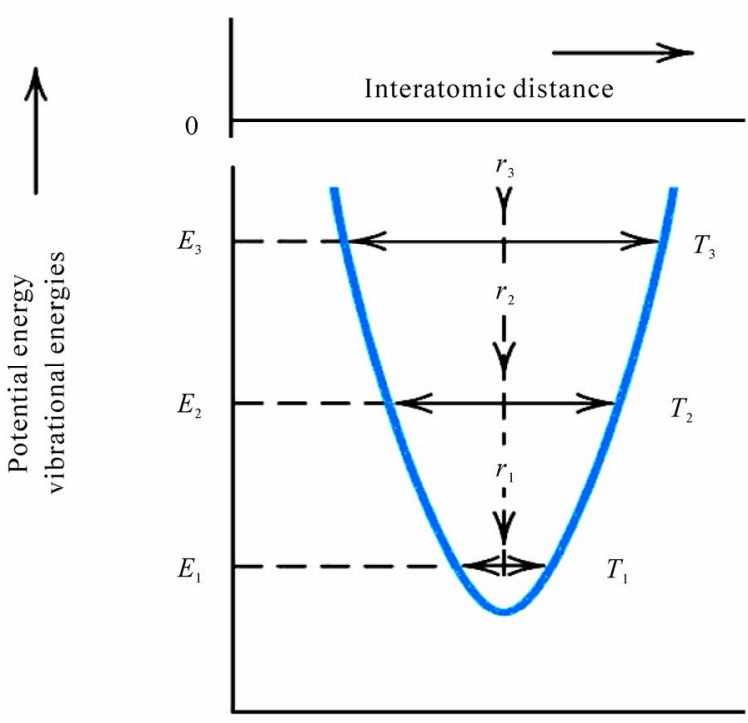

(b)

Figure 8. (a) Variation of asymmetric potential energy curve with Temperature T; (b) Variation of symmetric potential energy curve with Temperature $T$.

position, which increases with temperature from $\mathrm{r}\left(\mathrm{T}_{1}\right)$ to $\mathrm{r}\left(\mathrm{T}_{5}\right)$. Thermal expansion is really due to the asymmetric curvature of this potential energy trough, rather than the increased atomic vibration amplitudes with rising temperature. The coefficient of thermal expansion ' $\alpha$ ' is larger if $\mathrm{E}_{\mathrm{o}}$ is smaller and the curve is very asymmetric.

If the potential energy curve were symmetric (Figure 8(b)), there would be no net change in interatomic separation with rise of temperature and, consequently, no thermal expansion.

Magnitude of linear coefficient of thermal expansion $\alpha$ increases with increase in temperature. If inter-atomic energy is large, and the well of potential curve is deep and narrow the increase in inter atomic separation with rise of temperature is small, yielding a small $\alpha$ value. This is observed in materials having strong bondng energies. When the material has small bond energies inter atomic spacing increases with temperatue rise indicating high thermal expansion $\alpha$. (Figure 9). [2] 


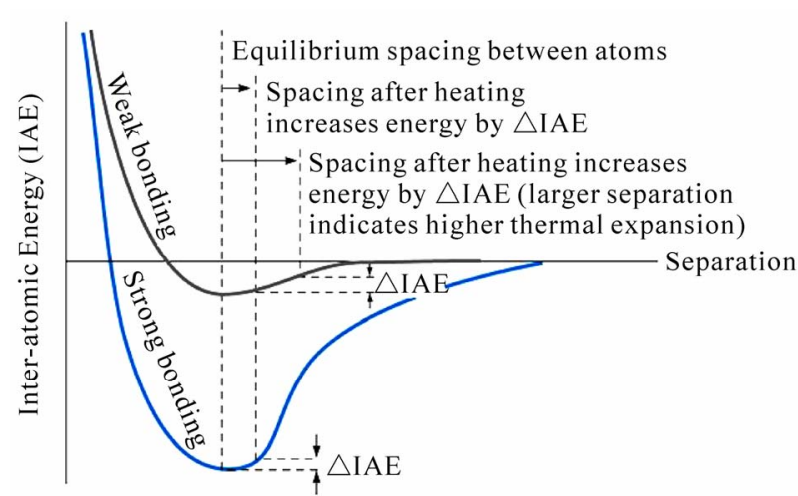

Figure 9. The inter-atomic energy separation curve for two atoms. Materials that display a steep curve with a deep trough have low linear coefficients of thermal expansion.

Thermal conductivity values are lower for polymers, intermediate for ceramics and maximum for metals. This is because in metals the vibration transfer is through atoms and electrons, in ceramics it is through atoms and in polymers it is due to rotation and vibration of long chain molecules.

\subsection{Electrical Properties}

Solid materials exhibit a very wide range of electrical conductivity $[1,3]$. Electrical conductivity and resistivity are material parameters which are geometry independent. The magnitude of electrical conductivity is strongly dependent on the number of electrons available to participate in the conduction process.

Consider a solid of $\mathrm{N}$ atoms. Initially, at relatively large separation distances, each atom is independent of its neighbors. However as the atoms approach close with one another electrons of one atom are perturbed by electron and nuclei of another. This influences each distinct atomic state to split into a series of closely spaced electronic levels in the solid termed as an electron energy band (Figure 10). Large numbers of individual energy levels overlap and form a band (Figure 11). The number of states within each band equals the total of all states contributed by the $\mathrm{N}$ atoms. Within each band, the energy states are discrete, yet the difference between adjacent states is exceedingly small. Furthermore, gaps may exist in the adjacent bands.

Four different types of band structures are possible at 0 K. In Figure 12(a) the outermost band is only partially filled with electrons. This type of band structure is seen in metals with a single $\mathrm{s}$ valence electron. For the second band structure in Figure 12(b) there is an overlap of an empty band and a filled band. In the last two band structures Figure 12(c) and Figure 12(d) the band completely filled with electrons is separated from an empty conduction band. The magnitude of energy gap is the

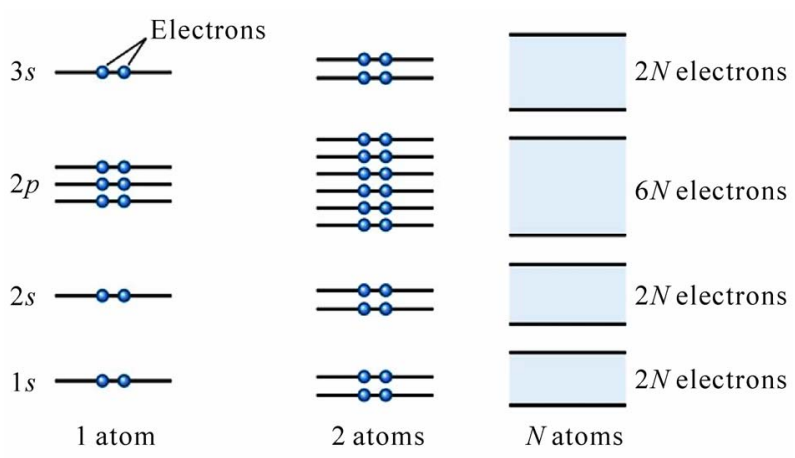

Figure 10. The energy levels broaden into bands as the number of electrons grouped together increases.
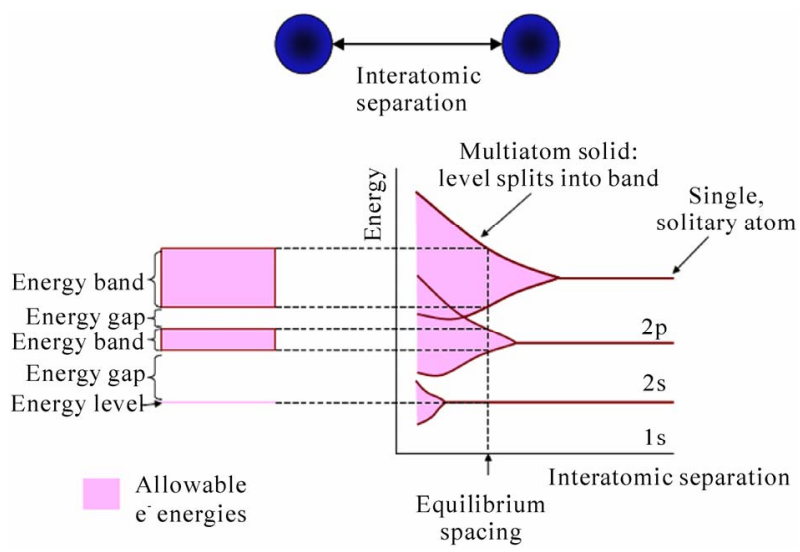

Figure 11. Energy band structure in solids.

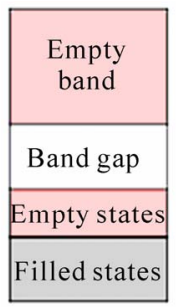

(a)

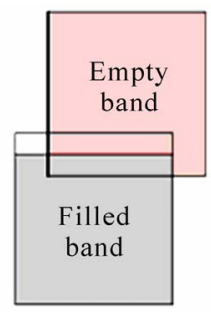

(b)

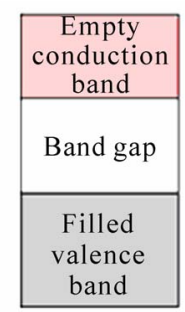

(c)

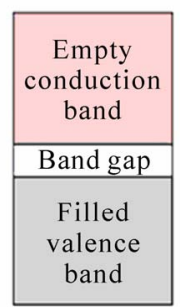

(d)
Figure 12. The various possible electron band structures in solids at $0 \mathrm{~K}$ : (a) Metals such as copper, in which electron states are available above and adjacent to filled states, in the same band; (b) The electron band structure of metals such as magnesium, wherein there is an overlap of filled and empty outer bands; (c) Insulators: the filled valence band is separated from the empty conduction band by a relatively large band gap ( $>2 \mathrm{eV})$; (d) Semiconductors: same as for insulators except that the band gap is relatively narrow ( $<2 \mathrm{eV})$.

only difference between the two band structures, for materials that are insulators the band gap is wide whereas for semiconductors it is narrow [1].

Conductors, semiconductors, and insulators have different accessibility to energy states for conductance of 
electrons. Metallic conductivity is of the order of $10^{7}$ $(\Omega-m)^{-1}$, semiconductors have intermediate conductivities $10^{-6}$ to $10^{4}(\Omega-\mathrm{m})^{-1}$ and insulators have very low conductivities $10^{-10}$ to $10^{-20}(\Omega-\mathrm{m})^{-1}$.

\section{Conclusions}

The magnitude of bonding energy and shape of the potential energy curve varies from material to material. A deep and narrow trough in the curve indicates large bond energy, high melting temperature, large elastic modulus and small coefficient of thermal expansion.

Generally substances with large bonding energy $E_{o}$ are solids, intermediate energies are liquids and small energies are gases.

The width and asymmetry of the well in the potential energy curve represents varying properties of different materials.

Large bond energies, high melting temperature, large elastic modulus and small coefficient of linear expansion found in potential energy curve(s) of ceramics represent very good strength, characteristically hard nature.

The potential energy curves of metals possess variable bond energy, reasonably high melting point, high elastic modulus, and moderate thermal expansion. The ductility of metals is implicitly related to the characteristics of the metallic bond. The grouping of energy levels as bands and their overlap with availability of large number of free electrons is responsible for electrical conductivity of metals.
The potential curve(s) of polymers possess low melting point low elastic modulus and large coefficient of linear expansion. Polymers possessing directional properties due to covalent bonding have dominating seconddary forces of interactions and influence the physical properties of materials.

This paper focused on an ideal situation involving only two atoms to understand some of the properties, a similar yet more complex condition exists for solid materials as interactions among many atoms need to be addressed. However, the energy versus interatomic separation curve defines the basic property.

In many materials more than one type of bonding is involved viz., ionic and covalent in ceramics, covalent and secondary in polymers, covalent and ionic in semiconductors. These are to be considered while deriving the properties from the potential energy curves.

\section{REFERENCES}

[1] W. D. Callister, "Materials Science \& Engineering: An Introduction," 7th Edition, John Wiley \& Sons, Inc., Hoboken, 2008.

[2] D. R. Askeland and P. P. Phule. "The Science and Engineering of Materials," 4th Edition, Thomas Book Company, Pacific Grove, 2003.

[3] W. F. Smith, "Principles of Materials Science and Engineering," 3rd Edition, McGraw-Hill, Columbus, 1996. 